\title{
Radio Continuum Surveys and Galaxy Evolution: Modelling and Simulations
}

\author{
A. Slyz ${ }^{1 *}$, J. Devriendt ${ }^{1}$, M. Jarvis ${ }^{1,2}$, Y. Dubois ${ }^{3,4}$ and C. Pichon ${ }^{3,4}$ \\ ${ }^{1}$ University of Oxford, Astrophysics, Denys Wilkinson Building, Keble Road, OX1 3RH, UK \\ ${ }^{2}$ Physics Department, University of Western Cape, Bellville 7535, South Africa \\ ${ }^{3}$ Sorbonne Universités, UPMC Univ Paris 06, UMR 7095, F-75005, Paris, France \\ ${ }^{4}$ CNRS, UMR 7095, Institut d'Astrophysique de Paris, $98^{\text {bis }}$ Blvd Arago, F-75014, Paris, France \\ E-mail: adrianne.slyz@physics.ox.ac.uk, \\ julien.devriendt@physics.ox.ac.uk, matt.jarvis@physics.ox.ac.uk, \\ dubois@iap.fr, pichoneiap.fr
}

We predict the evolution of the radio continuum sky at $1.4 \mathrm{GHz}$ from the Horizon-AGN Adaptive Mesh Refinement (AMR) cosmological hydrodynamical simulation of a cubic volume of the Universe $100 \mathrm{~h}^{-1} \mathrm{Mpc}$ on a side. With empirically motivated models for the radio continuum emission due to both star formation and Active Galactic Nuclei (AGN), we estimate the contribution of each of these processes to the local radio continuum luminosity function (LF) and describe its evolution up to redshift 4 . Despite the simplicity of these models, we find that our predictions for the local luminosity function are fairly consistent with Mauch \& Sadler (2007) observations, with the faint end of the luminosity function dominated by star forming galaxies and the bright end by radio loud AGNs. At redshift one, a decent match to Smolcic et al. (2009) VLA data in the COSMOS field can only be achieved when we account for radio continuum emission from AGNs. We predict that the strongest evolution across the peak epoch of cosmic activity happens for low luminosity star forming galaxies $L_{1.4 \mathrm{GHz}}<10^{22} \mathrm{~W} \mathrm{~Hz}^{-1}$, whose contribution rises until $z \sim 2$ and declines at higher redshifts. The contribution of low luminosity AGNs $L_{1.4 \mathrm{GHz}}<10^{22} \mathrm{~W} \mathrm{~Hz}^{-1}$ steadily declines from $z=0$ throughout the redshift range, whilst that of radio loud objects with luminosities in the range $10^{22} \mathrm{~W} \mathrm{~Hz}^{-1}<L_{1.4 \mathrm{GHz}}<10^{24} \mathrm{~W} \mathrm{~Hz}^{-1}$ rises dramatically until $z=4$. Finally, high-luminosity radio loud AGNs, with $L_{1.4 \mathrm{GHz}}>10^{24} \mathrm{~W} \mathrm{~Hz}^{-1}$ show surprisingly little evolution from $z=0$ to $z=4$.

The many facets of extragalactic radio surveys: towards new scientific challenges (*)

20-23 October 2015

Bologna, Italy

(*) This conference has been organized with the support of the Ministry of Foreign Affairs and International Cooperation, Directorate General for the Country Promotion (Bilateral Grant Agreement ZA14GR02 - Mapping the Universe on the Pathway to SKA)

* Speaker. 


\section{Introduction}

Simulations of the extragalactic radio continuum sky have been largely restricted to a semiempirical approach where simulated sources are drawn at random from an observed or extrapolated radio continuum LF. The most sophisticated of these simulations produced as part of the SKA Design Study (Wilman et al. 2008), accounts for the clustering of the radio continuum sources on large scales by grafting them onto an underlying dark matter density field which evolves under linear theory. A range of sources (radio quiet, radio loud divided into Fanaroff-Riley type I (FR I) and FR II classes, quiescent star forming galaxies and starburst galaxies) are modelled as simple structures: point sources and ellipses. The great advantage of a simulation based on a semi-empirical approach is that it can map large fields of view, something out of reach of current large volume hydrodynamical cosmological simulations. The limitations however are sizeable and include a limited predictive power for the evolution of radio continuum sources because of the use of extrapolated LFs. In a semi-empirical approach individual galaxies do not undergo changes. There are no galaxies that are sometimes star forming, sometimes active AGN galaxies, and sometimes hybrids.

Provided a credible model for the radio continuum emission can be found, a cosmological hydrodynamical simulation can address these limitations. In this vein, we use the Horizon-AGN simulation to explore predictions for the radio continuum LF given physically motivated, observationally calibrated prescriptions linking a galaxy's star formation rate and black hole accretion rate to its radio continuum emission. Although covering only about a tenth of the sky covered by the Wilman et al. 2008 radio continuum sky simulation and falling short of modelling ab initio the complex physics thought to be responsible for radio continuum emission (e.g. magnetic fields, radiative transfer), the Horizon-AGN hydrodynamical cosmological simulation offers advantages over previous semi-empirical approaches (e.g. Hopkins et al. 2000, Windhorst 2003, Jarvis \& Rawlings 2004, Wilman et al. 2008). These include a modelling of the non-linear evolution of the dark matter density field, a better modelling of gas outflows, inflows and their interaction on the scales of the intergalactic medium, a description of the interaction between galaxies, the natural occurrence of galaxies that are both star forming and hosts of black holes with star formation or AGN activity dominating their emission to different extents at different points in their histories. These advantages allow us to predict the evolution of the LF rather than rely on extrapolations of the redshift zero LF.

\section{The Horizon-AGN simulation}

We give a brief description of the Horizon-AGN simulation (Dubois et al., 2014), a large volume cosmological hydrodynamical simulation, run with the Adaptive Mesh Refinement code, RAMSES (Teyssier, 2002). It adopts a standard $\Lambda$ CDM cosmology with total matter density $\Omega_{m}=$ 0.272 , dark energy density $\Omega_{\Lambda}=0.728$, amplitude of the matter power spectrum $\sigma_{8}=0.81$, baryon density $\Omega_{b}=0.045$, Hubble constant $H_{0}=70.4 \mathrm{~km} \mathrm{~s}^{-1} \mathrm{Mpc}^{-1}$, and $n_{s}=0.967$ compatible with a WMAP-7 cosmology (Komatsu et al., 2011). The size of the box is $L_{b o x}=100 h^{-1} \mathrm{Mpc}$ with $1024^{3} \mathrm{DM}$ particles, which gives a dark matter (DM) mass resolution of $M_{D M}=8 \times 10^{7} \mathrm{M}_{\odot}$.

A cell is refined up to an effective resolution of $\Delta x=1 \mathrm{kpc}$ (physical) according to a quasiLagrangian criterion: if the mass in a cell is more than 8 times that of the initial mass resolution, 
another refinement level is added. The simulation include prescriptions for background UV heating, gas cooling including the contribution from metals released by stellar feedback, star formation following a Schmidt law with a $1 \%$ efficiency (Rasera \& Teyssier, 2006), star formation density threshold of $n_{0}=0.1 \mathrm{H} \mathrm{cm}^{-3}$ and feedback from stellar winds and type Ia and type II supernovae assuming a Salpeter initial mass function (IMF) (Dubois \& Teyssier 2008, Kimm 2012).

Black holes (BHs) are created in cells where the combined gas and stellar density exceeds the threshold for star formation $\left(n_{0}=0.1 \mathrm{H} \mathrm{cm}^{-3}\right)$, and where the stellar velocity dispersion in that cell is larger than $100 \mathrm{~km} \mathrm{~s}^{-1}$, with an initial seed mass of $10^{5} \mathrm{M}_{\odot}$. In order to avoid the formation of multiple BHs in the same galaxy, BHs are not allowed to form at distances smaller than 50 comoving kpc from any other BH particle. BHs grow through accretion and mergers with other black holes. The accretion rate onto BHs follows the Bond-Hoyle-Littleton rate multiplied by a dimensionless boost factor $\alpha=\left(\rho / \rho_{0}\right)^{2}$ when $\rho>\rho_{0}$ and $\alpha=1$ otherwise (Booth \& Schaye 2009 ) in order to account for our inability to capture the colder and higher density regions of the ISM. The effective accretion rate onto BHs is capped at the Eddington luminosity with an assumed radiative efficiency of $\varepsilon_{r}=0.1$ for the Shakura \& Sunyaev (1973) accretion onto a Schwarzschild BH.

The AGN feedback is a combination of two different modes, the so-called radio mode operating when $\chi=\dot{M}_{B H} / \dot{M}_{\text {Edd }}<0.01$ and the quasar mode otherwise. The quasar mode corresponds to an isotropic injection of thermal energy into the gas within a sphere of radius $\Delta x$, at an energy deposition rate: $\dot{E}_{A G N}=\varepsilon_{f} \varepsilon r \dot{M}_{B H} c^{2}$, where $\varepsilon_{f}=0.15$ for the quasar mode is a free parameter chosen to reproduce the correlations between BHs and galaxies and the BH density in our local Universe (see Dubois et al., 2012). At low accretion rates, on the other hand, the radio mode deposits the AGN feedback energy into a bipolar outflow with a jet velocity of $10^{4} \mathrm{~km} / \mathrm{s}$ into a cylinder with a cross section of radius $\Delta x$ and height $2 \Delta x$ following Omma et al. (2004) (more details about the jet implementation are given in Dubois et al., 2010). The efficiency of the radio mode is larger than the quasar mode, with $\varepsilon_{f}=1$.

The success of the Horizon-AGN simulation in reproducing stellar mass functions, the cosmic star formation rate density, and the stellar main sequence is provided in Kaviraj et al. in prep. Details of how well the simulation reproduces BH and AGN properties are presented in Volonteri et al. (2016). Figure 1 displays a slice through the Horizon-AGN volume at redshift 0.5, giving an idea of the large statistical sample the simulation provides (about 150,000 galaxies per snapshot out to redshift two, 109,060 galaxies at redshift three, and 44,153 galaxies at redshift four).

\section{Modelling radio continuum}

\subsection{Contribution from star forming galaxies}

A tight correlation between far-infrared (FIR) and radio continuum emission in local galaxies is attributed to massive star formation (Harwit \& Pacini, 1975; Yun, Reddy \& Condon 2001). Although the details of the link are not fully understood, the idea is that FIR emission comes from hot dusty envelopes around massive stars, while radio continuum emission, divided into a thermal and a non-thermal component, also ultimately originates from massive stars. HII regions 


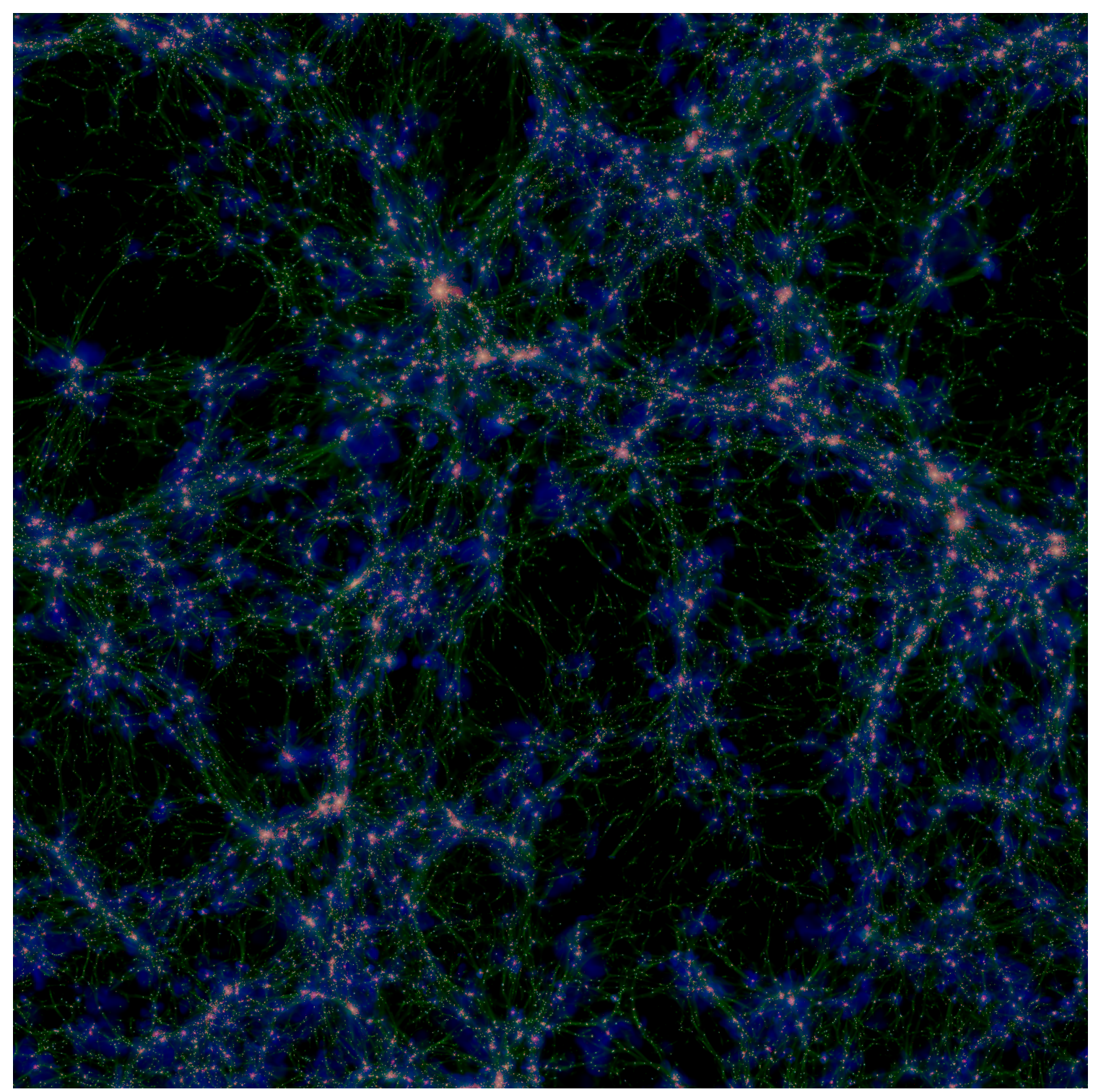

Figure 1: A three color image of a slice through the Horizon-AGN simulation at redshift 0.5. The slice shows the full height and width of the simulation volume $\left(100 \mathrm{~h}^{-1} \mathrm{Mpc}\right)$ and is projected over $20 \%$ of its depth. Green corresponds to gas density, blue to metallicity and red to stars.

around massive stars shine in free-free emission dominating the thermal radio continuum emission, while relativistic electrons released and accelerated during supernovae explosions from the same population of massive stars are thought to be the main source of the non-thermal radio continuum emission. Widely observed for local star forming galaxies, VLA $1.4 \mathrm{GHz}$ and MIPS 24 and 70 $\mu \mathrm{m}$ observations of the $2 \mathrm{deg}^{2}$ COSMOS field combined with an extensive multiwavelength data set give firm support to previous findings (Garrett, 2002; Appleton et al., 2004; Frayer et al., 2006) that the FIR-radio relation holds out to at least redshift $\sim 1.4$ and most likely also holds between redshift 2.5 and 5 (Sargent et al., 2010).

Independent of the redshift of our simulation, we therefore adopt the simple model for radio 
emission due to star formation from Condon (1992). It depends on the star formation rate, $\dot{M}_{\star}$, for stars more massive than $5 \mathrm{M}_{\odot}$ according to the following relations:

$$
\begin{gathered}
\left(\frac{L_{\text {non-thermal }}}{W H z^{-1}}\right) \sim 5.3 \times 10^{21}\left(\frac{v}{G H z}\right)^{-0.8}\left(\frac{\dot{M}_{\star}\left[M \geq 5 M_{\odot}\right]}{M_{\odot} y r^{-1}}\right) \\
\left(\frac{L_{\text {thermal }}}{W H z^{-1}}\right) \sim 5.5 \times 10^{20}\left(\frac{v}{G H z}\right)^{-0.1}\left(\frac{\dot{M}_{\star}\left[M \geq 5 M_{\odot}\right]}{M_{\odot} y r^{-1}}\right)
\end{gathered}
$$

where $v$ is the frequency of emission. Our measurement of $\dot{M}_{\star}$ is averaged over a period of 100 Myr in the simulation.

For the purposes of the supernova feedback, the Horizon-AGN simulation assumes a Salpeter IMF, but nothing prevents us from giving a prediction for the radio continuum emission for the case where stars form with a different IMF. Fig. 2 shows Horizon-AGN's prediction for the 1.4 GHz LF due to star formation only. The left panel assumes the Salpeter IMF with lower and upper mass cutoffs of $M_{\text {low }}=0.1 \mathrm{M}_{\odot}$ and $M_{\text {up }}=100 \mathrm{M}_{\odot}$ respectively. The right hand panel gives the prediction using the Chabrier IMF. Mass cutoffs are the same as for the case with the Salpeter IMF, but now we see that because for the same amount of stars formed the Chabrier IMF produces a greater number of massive stars than the Salpeter IMF, the entire LF is shifted to higher luminosities with the Chabrier IMF, better matching the high luminosity end of the observed LF which we take from Mauch \& Sadler 2007. These observations comprise $\sim 8000$ radio sources from the $1.4 \mathrm{GHz}$ NRAO VLA Sky Survey (NVSS) associated with galaxies brighter than K $=12.75$ mag in the Second Incremental Data Release of the 6 degree Field Galaxy Survey (6dFGS DR2). 6dF spectra for the galaxies allow a classification of the galaxy sample by dominant radio emission mechanism, i.e. star formation versus AGN powered by a supermassive black hole.

An advantage of predicting the radio continuum from a cosmological hydrodynamical simulation is that one can predict the LF at higher redshifts, rather than extrapolating zero redshift LFs. Fig. 3 shows that the LF evolves in line with the evolution seen in the cosmic star formation rate density. To facilitate a comparison with semi-empirical models, we also plot on the figure the LF we would infer from the simulation assuming pure luminosity evolution of the $z=0$ galaxy population. In other words, we also represent $\Phi_{z}(L)=\Phi_{z=0}\left[L /(1+z)^{\alpha_{L}}\right]$, for different values of $\alpha_{L}$, alongside with $\Phi_{z}(L)$ as directly measured in the simulation. We conclude that for redshifts $z<3$ the low luminosity part of the LF shifts to higher luminosities with $\alpha_{L}=2$, while the high luminosity part of the LF has a slower evolution, with luminosities only increasing in line with $\alpha_{L}=1$. From redshift 3 onwards, in synch with the downturn in the cosmic star formation rate density, the $1.4 \mathrm{GHz}$ LF due to star forming galaxies in the simulation shifts to lower luminosities. We plot the redshift one VLA-COSMOS data for star forming galaxies in Smolcic et al. 2009 and find that if we only consider radio continuum predicted from star formation, the simulation vastly under predicts these observations (by either an order of magnitude in luminosity or two orders of magnitude in number density), but note that at redshift one the VLA-COSMOS data is picking up extremely bright and rare galaxies, e.g. ULIRGS and even HyLIRGS (Smolcic et al. 2008) which are in deficit in the Horizon-AGN simulation due to the small box size $\left(100 \mathrm{~h}^{-1} \mathrm{Mpc}\right)$, and very likely to suffer from a significative contribution of a central AGN to their radio flux. 

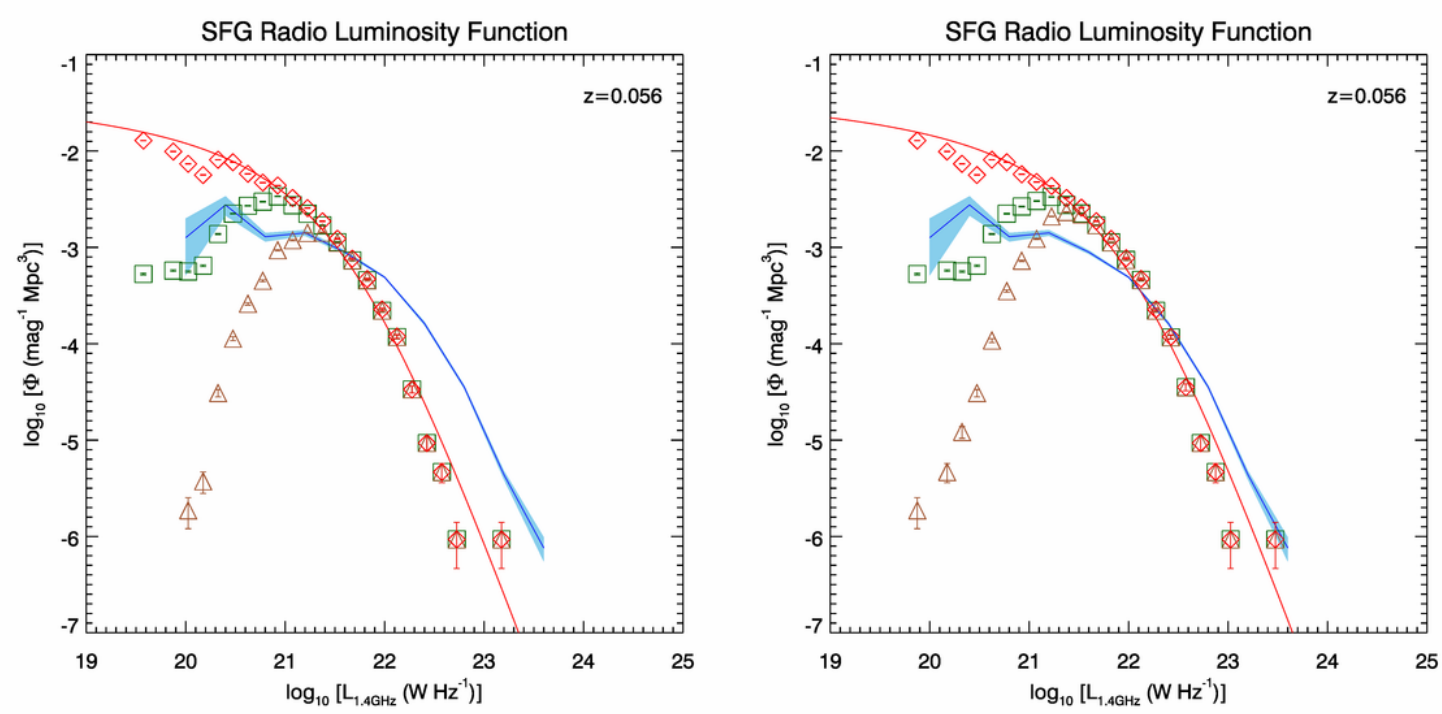

Figure 2: Star forming contribution to the $1.4 \mathrm{GHz}$ radio LF predicted from the Horizon-AGN simulation (symbols) at $\mathrm{z}=0.056$ and compared to the Mauch \& Sadler 2007 observations for the contribution of local star forming galaxies (SFG) to the $1.4 \mathrm{GHz}$ LF (blue solid line with error bars indicated in blue shading). Red diamonds show the LF for all galaxies in the Horizon-AGN for which the simulation is complete $\left(M_{\star} \geq\right.$ $2 \times 10^{8} \mathrm{M}_{\odot}$ ); green squares show the LF only for galaxies in the simulation with $M_{\star} \geq 10^{10} \mathrm{M}_{\odot}$ and brown triangles show the LF for all galaxies in the simulation more massive than $10^{10} \mathrm{M}_{\odot}$ and with flux greater than $10 \mathrm{mJy}$. The left panel shows the simulation results when the Salpeter IMF is assumed and the right panel shows the results when the Chabrier IMF is assumed.

\subsection{Contribution from AGN}

While the FIR-radio correlation is tight for star forming galaxies, galaxies with strong AGN signatures tend to show a radio excess in this correlation (e.g. Mauch \& Sadler, 2007) indicating a different mechanism for exciting radio continuum emission. In this work, we exploit the analogy between X-ray binaries and AGNs to make predictions for the radio continuum of active galaxies. More specifically, we assume that with their flat radio spectra and compact jets, hard state X-ray binaries are analogous to low-luminosity AGN which accrete at low Eddington rates and are also observed to have a flat spectrum radio core and jets. On the other hand, with their unstable jets and propensity to eject highly relativistic blobs of material, intermediate state X-ray binaries are taken to be linked to radio loud AGNs which accrete at high Eddington rates and release energy in extended radio lobes.

Unlike observers who deduce the mass accretion rate onto X-ray binaries from their luminosities, we measure the mass accretion rate onto black holes in the Horizon-AGN simulation, but need a prescription to relate this mass accretion rate to the radio luminosity. We use two different prescriptions depending on whether $\chi=\dot{M}_{\mathrm{BH}} / \dot{M}_{\text {Edd }}$ is high or low. For low accretion rates $\left(\chi<\chi_{\text {low }}\right)$, we assume a low luminosity AGN and therefore use the relation between the core radio luminosity, $L_{\text {rad }}$ in eq. 3.3, and mass accretion rate for hard state objects (Körding, Fender, \& Migliari 2006):

$$
\dot{M}\left(g^{-1}\right) \sim 4 \times 10^{17}\left(\frac{L_{\text {rad }}}{10^{30} \operatorname{erg~s}^{-1}}\right)^{12 / 17} .
$$



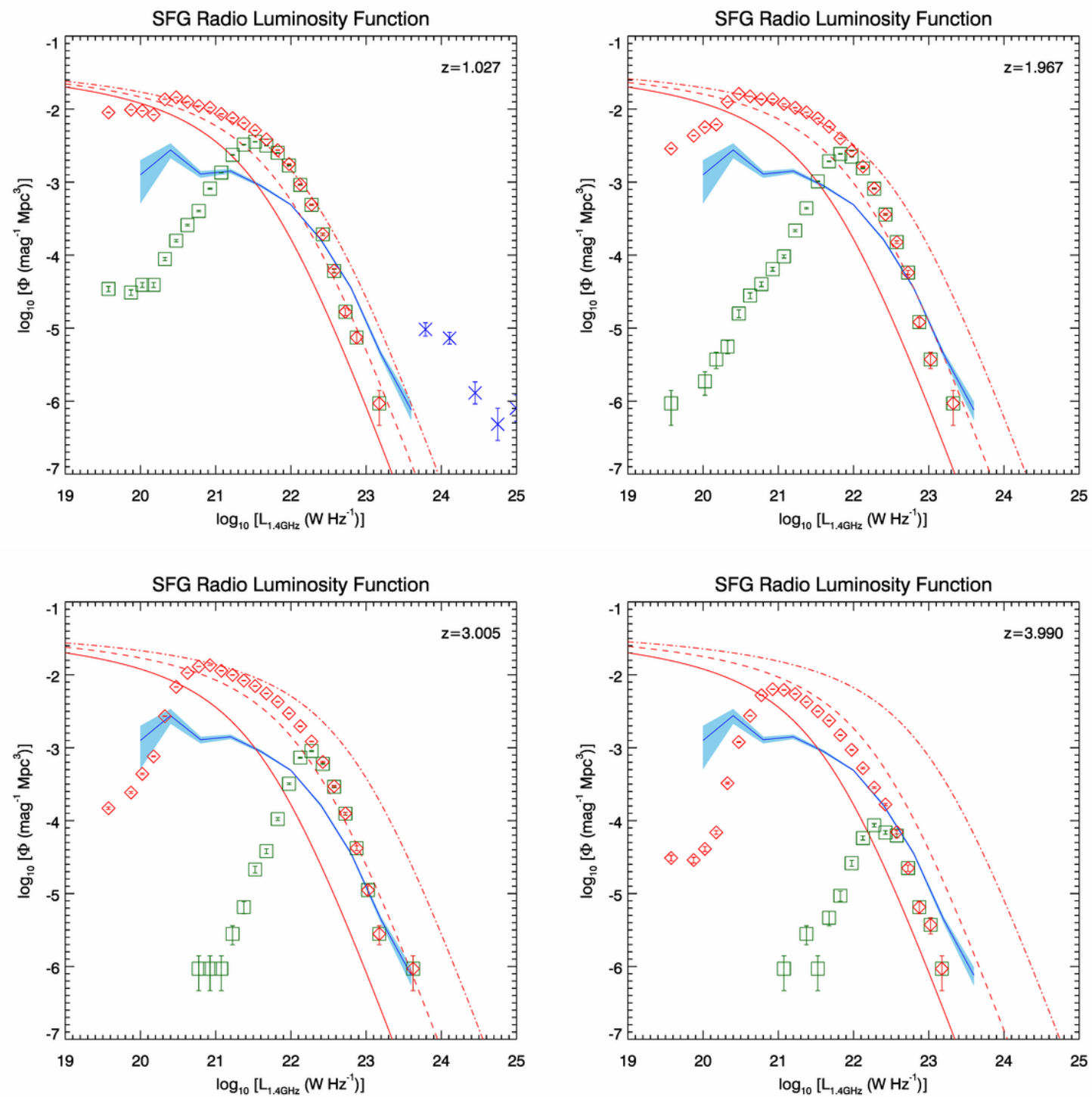

Figure 3: Evolution of the $1.4 \mathrm{GHz}$ radio $\mathrm{LF}$ predicted from the star formation rates of galaxies in the Horizon-AGN simulation at (top left) $\mathrm{z}=1.027$, (top right) $\mathrm{z}=1.967$, (bottom left) $\mathrm{z}=3.005$,. and (bottom right) $\mathrm{z}=3.990$. All predictions are given with the Salpeter IMF. As in Fig. 2, Mauch \& Sadler 2007 observations for local star forming galaxies are represented by a blue solid line with error bars indicated in blue shading. Red diamonds show the LF for all galaxies in the Horizon-AGN for which the simulation is complete $\left(M_{\star} \geq 2 \times 10^{8} \mathrm{M}_{\odot}\right)$; green squares show the LF only for galaxies in the simulation with $M_{\star} \geq$ $10^{10} \mathrm{M}_{\odot}$. The red solid line indicates the Horizon-AGN prediction for the $1.4 \mathrm{GHz} \mathrm{LF}$ at $\mathrm{z}=0.056$. The red dashed line shows the result of scaling Horizon-AGN's redshift $0.056 \mathrm{LF}$ assuming pure luminosity evolution with $\alpha_{L}=1$ (see text for definition) and the red dot-dash line is for the same assumption but with a value $\alpha_{L}=2$. Large blue asterices show redshift one VLA-COSMOS data from Smolcic et al. 2009. 

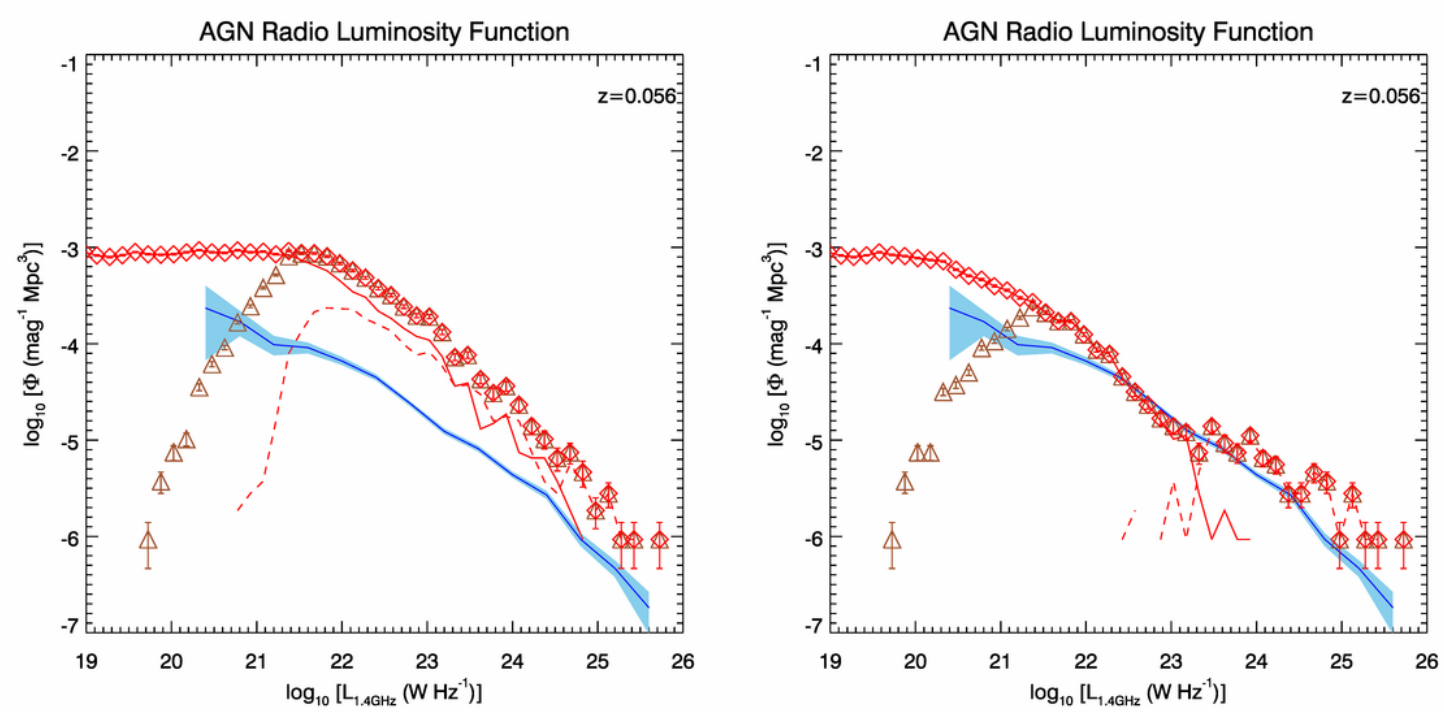

Figure 4: AGN contribution to the $1.4 \mathrm{GHz}$ radio LF predicted from the Horizon-AGN simulation (symbols) at $\mathrm{z}=0.056$ compared to the Mauch \& Sadler 2007 observations of the $1.4 \mathrm{GHz} \mathrm{LF}$ for radio-loud AGNs (blue solid line with error bars indicated in blue shading). Red diamonds show the LF for all galaxies in the Horizon-AGN for which the simulation is complete $\left(M_{\star} \geq 2 \times 10^{8} \mathrm{M}_{\odot}\right)$; brown triangles show the LF for all galaxies in the simulation more massive than $10^{10} \mathrm{M}_{\odot}$ and with flux greater than $10 \mathrm{mJy}$. The left panel shows the simulation results when $\chi_{\text {low }}=\chi_{\text {high }}=0.01$ whereas the right panel shows the results when $\chi_{\text {low }}=0.001$ and $\chi_{\text {high }}=0.3$. The solid red line through the symbols denotes the contribution from lowluminosity AGN, whereas the dashed red line through the symbols indicates the contribution from radio-loud AGN.

Assuming a flat spectrum for the radio luminosity, $L_{\mathrm{rad}} \sim v L_{v}$, we solve for the radio luminosity at $1.4 \mathrm{GHz}$.

For high accretion rates $\left(\chi>\chi_{\text {high }}\right)$, we reason that we are in the regime of radio loud AGN, and therefore use the correlation between the extended radio emission at $151 \mathrm{MHz}$ ( $L_{151}$ in eq. 3.4) and the mass accretion rate for intermediate state sources (Körding, Jester \& Fender 2008):

$$
\dot{M}\left(g s^{-1}\right) \sim \log L_{151}\left(W H z^{-1} s r^{-1}\right)-0.15 .
$$

Further assuming a steep spectrum for the radio luminosity typical of these sources, i.e. $L_{v} \sim v^{-0.7}$, allows us to convert their luminosity at $151 \mathrm{MHz}$ to that at $1.4 \mathrm{GHz}$.

We find that the cutoffs between what we consider high and low accretion rates significantly impact the predicted LF. Choosing the same cutoff for the LF modelling that we use for the AGN feedback, namely $\chi_{\text {low }}=\chi_{\text {high }}=0.01$, leads to an overprediction by as much as one order of magnitude in the LF (left panel of Fig. 4). We are able to curb this excess of sources by allowing $\chi_{\text {low }}$ and $\chi_{\text {high }}$ to vary as free parameters. The right panel of Fig. 4 displays results for $\chi_{\text {low }}=0.001$ and $\chi_{\text {high }}=0.3$. Alternatively, one could keep the fiducial value of $\chi_{\text {low }}=\chi_{\text {high }}=0.01$ and assume that only about $10 \%$ of the entire AGN population is radio loud, which seems consistent with observations (e.g. White et al, 2000).

The upper left panel of fig. 5 shows that accounting for radio loud AGN in the prediction for the 1.4 GHz LF gives a better match to the redshift one VLA-Cosmos data (Smolcic et al. 2009) than 

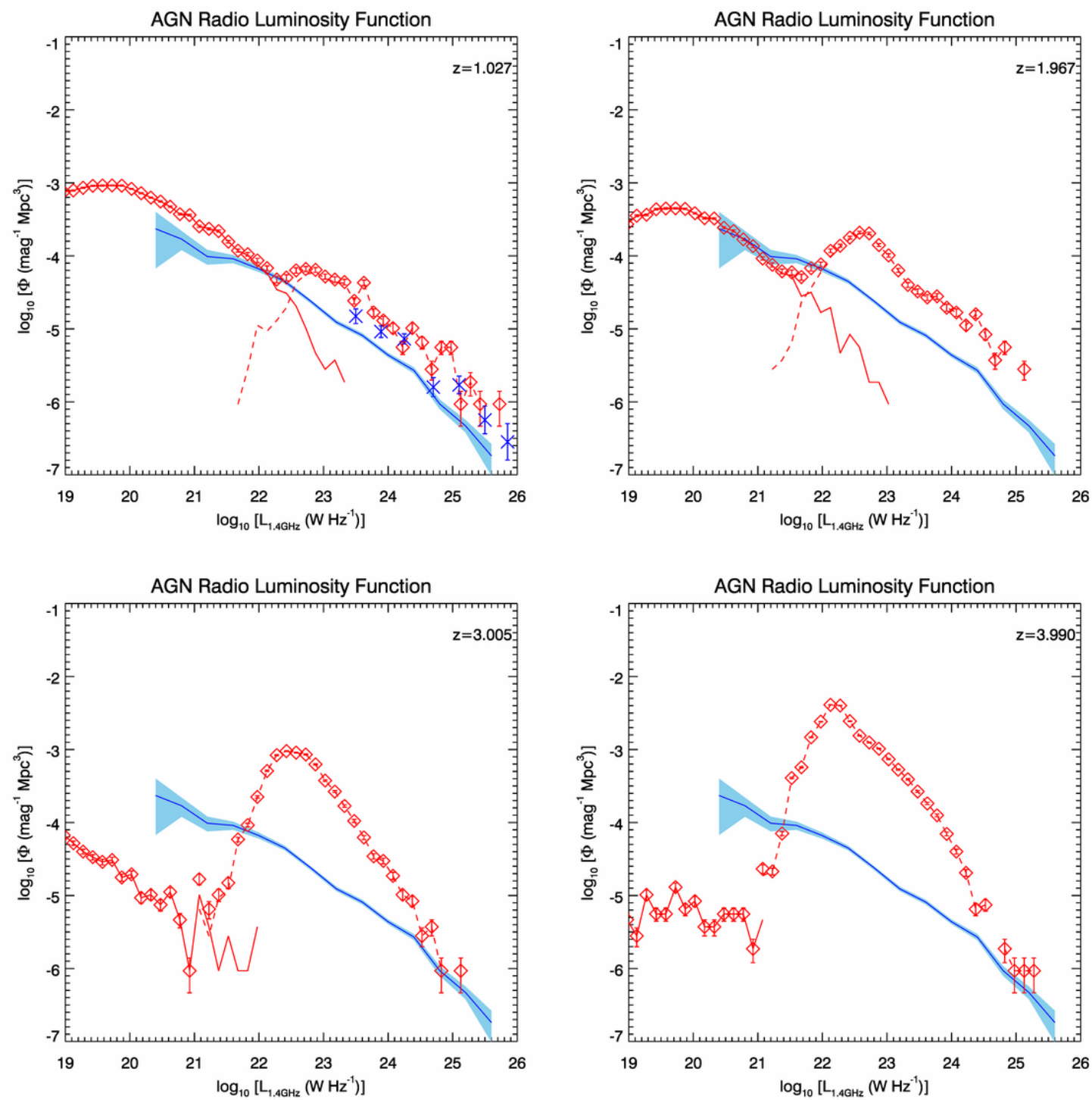

Figure 5: Evolution of the AGN contribution to the 1.4 GHz radio LF for galaxies in the Horizon-AGN simulation at (top left) $\mathrm{z}=1.027$, (top right) $\mathrm{z}=1.967$, (bottom left) $\mathrm{z}=3.005$, and (bottom right) $\mathrm{z}=3.990$. All predictions are given with the model where $\chi_{\text {low }}=0.001$ and $\chi_{\text {high }}=0.3$. As in Fig. 3, Mauch $\&$ Sadler 2007 observations for the $1.4 \mathrm{GHz}$ LF for radio-loud AGNs are represented by a blue solid line with error bars indicated in blue shading. Red diamonds show the LF for all galaxies in the Horizon-AGN for which the simulation is complete $\left(M_{\star} \geq 2 \times 10^{8} \mathrm{M}_{\odot}\right)$. The solid red line through the symbols denotes the contribution from low-luminosity AGN, whereas the dashed red line through the symbols indicates the contribution from radio-loud AGN. Large blue asterices show redshift one VLA-COSMOS data from Smolcic et al. 2009. 

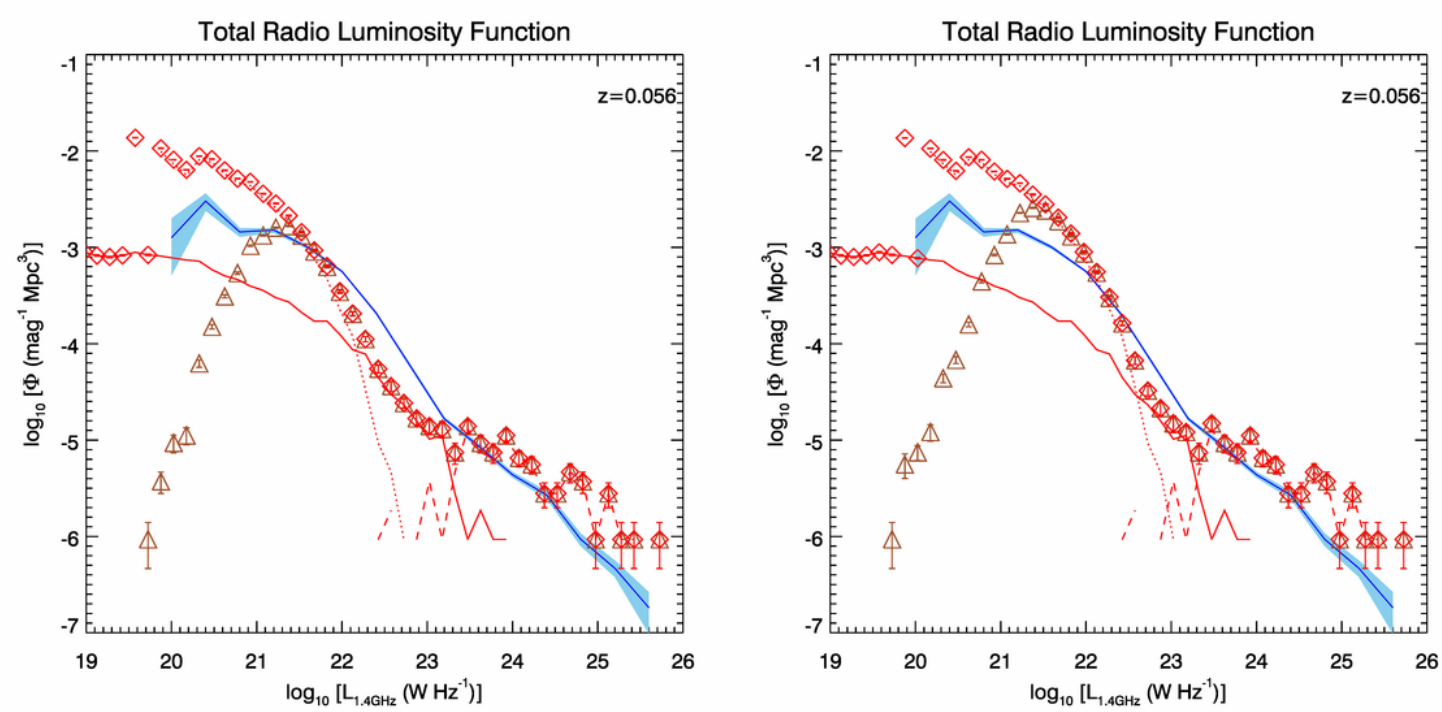

Figure 6: The Horizon-AGN prediction (symbols) at $\mathrm{z}=0.056$ for the $1.4 \mathrm{GHz}$ radio LF combining contributions to the radio continuum from both star formation and AGN. The Mauch \& Sadler 2007 observations for the $1.4 \mathrm{GHz} \mathrm{LF}$ for both star forming galaxies and radio-loud AGNs are represented by a blue solid line with error bars indicated in blue shading. Red diamonds show the LF for all galaxies in the Horizon-AGN for which the simulation is complete $\left(M_{\star} \geq 2 \times 10^{8} \mathrm{M}_{\odot}\right)$; brown triangles show the LF for all galaxies in the simulation more massive than $10^{10} \mathrm{M}_{\odot}$ and with flux greater than $10 \mathrm{mJy}$. The left (right) panel shows the simulation results when the Salpeter (Chabrier) IMF is assumed. Both panels use the model where $\chi_{\text {low }}=0.001$ and $\chi_{\text {high }}=0.3$ for the contribution from AGN to the radio continuum. The dotted red line through the symbols indicates the contribution to the LF from star formation, the solid red line denotes the contribution from low-luminosity AGN, whereas the dashed red line indicates the contribution from radioloud AGN.

the star formation contribution alone (upper left panel of fig. 3). Given that black hole accretion rates are generally higher at higher redshifts (before black holes self-regulate through feedback), fig. 5 naturally shows that the radio loud AGN contribution to the LF monotonically grows with increasing redshift while the low-luminosity AGN contribution steadily declines. Note that the bulk of the evolution occurs for radio loud AGNs with luminosities $10^{22} \mathrm{~W} \mathrm{~Hz}^{-1}<L_{1.4 \mathrm{GHz}}<10^{24} \mathrm{~W}$ $\mathrm{Hz}^{-1}$ and that it shows no sign of decline even at $z=4$. On the other hand, the highest luminosity radio loud AGNs in the simulation $\left(L_{1.4 \mathrm{GHz}}>10^{24} \mathrm{~W} \mathrm{~Hz}^{-1}\right)$ show little sign of evolution.

\section{Conclusion}

Despite the simple empirical calibration we used to estimate radio continuum emission from star formation and $\mathrm{BH}$ accretion rates, the Horizon-AGN simulation gives a reasonable match (fig. 6) to the observed local 1.4 GHz LF (Mauch \& Sadler 2007). As in the observations, star forming galaxies dominate the LF at low luminosities and radio-loud AGNs dominate at high luminosities. The choice of the IMF changes the prediction of the radio continuum emission fueled by star formation, and there appears to be a need to suppress the number of both low luminosity and radio-loud AGNs in the simulations to get a reasonable agreement with the observations. We 
emphasise that predictions of the radio continuum are particularly difficult because the physics of the radio continuum is complex and only partially modelled in the simulation. We also have to measure differential quantities: star formation and black hole accretion rates, which are notoriously more difficult to capture than their integrated counterparts. Given these caveats, the level of accuracy reached by this first attempt to estimate the radio continuum from a large volume cosmological simulation is encouraging. Future observations with the SKA and its precursors will tell if the evolution we predict for the different contributions of star forming galaxies and AGNs to the radio continuum $\mathrm{LF}$ is plausible.

\section{References}

Appleton, P.N. et al., The far and midinfrared radio correlations in the Spitzer Extragalactic First Look Survey, ApJS, 154, 147-150, 2004

Condon, J.J., Radio emission from normal galaxies, ARAA, 30, 575-611, 1992

Dubois, Y. et al., Jet-regulated cooling catastrophe, MNRAS, 409, 985-1001, 2010

Dubois, Y. et al., Self-regulated growth of supermassive black holes by a dual jet-heating active galactic nucleus feedback mechanism: methods, tests and implications for cosmological simulations, MNRAS, 420, 2662-2683, 2012

Dubois, Y. et al., Dancing in the dark: galactic properties trace spin swings along the cosmic web, MNRAS, 444, 1453-1468, 2014

Dubois, Y. \& Teyssier, R., On the onset of galactic winds in quiescent star forming galaxies, $A \& A$, 477, 79-94, 2008

Frayer, D. T. et al., Spitzer 70 and $160 \mu \mathrm{m}$ observations of the extragalactic first look survey, ApJ, 131, 250-260, 2006

Garrett, M. A., The FIR/Radio correlation of high redshift galaxies in the region of $H D F-N, A \& A$, 384, L19-L22, 2002

Harwit, M. \& Pacini, F., Infrared galaxies - Evolutionary stages of massive star formation, ApJ, 200, L127-L129, 1975

Hopkins, A. M. et al., What will the next generation radio telescope detect at 1.4 GHz?, Exp. Astron., 10, 419 - 437, 2000

Jarvis M., Rawlings S., The accretion history of the universe with the SKA, New Astron. Rev., 48, 1173-1185, 2004

Kimm T., On the importance of feedback in the stream-fed high redshift Universe, PhD Thesis, 2012

Komatsu, E. et al., Seven year Wilkinson Microwave Anisotropy Probe (WMAP) Observations: Cosmological Interpretation, ApJS, 192, 1-47, 2011 
Körding, E. et al., Jet-dominated advective systems: radio and X-ray luminosity dependence on the accretion rate, MNRAS, 369, 1451-1458, 2006

Körding, E. et al., Measuring the accretion rate and kinetic luminosity function of supermassive black holes, MNRAS, 383, 277-288, 2008

Mauch T., Sadler E., Radio sources in the 6DFGS: local luminosity functions at $1.4 \mathrm{GHz}$ for starforming galaxies and radio loud AGN, MNRAS, 375, 931-950, 2007

Omma, H. et al., Heating cooling flows with jets, MNRAS, 348, 1105-1119, 2004

Rasera Y., Teyssier R., The history of the baryon budget. Cosmic logistics in a hierarchical universe., $A \& A, 445,1-27,2006$

Sargent, M.T. et al., The VLA-COSMOS perspective on the infrared-radio relation. I. New constraints on selection biases and the non-evolution of the infrared/radio properties of star-forming and active galactic nucleus galaxies at intermediate and high redshift, ApJS, 186, 341-377, 2010

Shakura, N. I., Sunyaev R. A., Black holes in binary systems. Observational appearance., A\&A, 24, 337-355, 1973

Smolcic, V. et al., A new method to separate star-forming from AGN galaxies at intermediate redshift: the submillijansky radio population in the VLA-COSMOS survey, ApJS, 177, 14-38, 2008

Smolcic, V. et al., Cosmic evolution of radio selected active galactic nuclei in the cosmos field, ApJ, 696, 24-39, 2009

Teyssier, R., Cosmological hydrodynamics with adaptive mesh refinement. A new high resolution code called RAMSES, A \& A, 385, 337-364, 2002

Volonteri, M. et al., The cosmic evolution of massive black holes in the Horizon-AGN simulation, submitted to MNRAS, arXiv:1602.01941

White, R. L. et al., The FIRST Bright Quasar Survey II. 60 Nights and 1200 Spectra Later, ApJSS, 126, 133-207, 2000

Wilman, R.J. et al., A semi-empirical simulation of the extragalactic radio continuum sky for next generation radio telescopes, MNRAS, 388, 1335-1348, 2008

Windhorst, R., The microJansky and nanoJansky population, New Astron. Rev., 47, 357-365, 2003

Yun, M.S., Reddy, N. A., Condon, J.J., Radio properties of infrared selected galaxies in the IRAS 2 Jy sample, ApJ, 554, 803-822, 2001 